\title{
Enhanced Frequency Diversity Exploitation in Carrier Aggregation for LTE-Advanced Systems
}

\author{
Wenxun Qiu, Hlaing Minn \\ University of Texas at Dallas \\ Richardson, TX 75083 USA \\ qxun@student.utdallas.edu, hlaing.minn@utdallas.edu
}

\author{
Chia-Chin Chong \\ DOCOMO Communications Laboratories USA, Inc. \\ 3240 Hillview Avenue, Palo Alto, CA 94304 USA \\ cchong@docomolabs-usa.com
}

\begin{abstract}
LTE-Advanced systems have adopted the concept of Carrier Aggregation (CA) to meet the data rate requirements of IMT-Advanced systems. The CA approach opens up several new implementation challenges as well as opportunities for further performance improvement. Existing works on CA focus on implementation issues, but exploitation of CA-specific features for performance improvement has not been addressed yet. In this paper, we fill this gap and investigate the enhanced frequency diversity exploitation in $\mathrm{CA}$ based systems. New interleaving schemes for both downlink OFDMA and uplink SC-FDMA of LTE-Advanced systems are proposed to capture CA-specific enhanced frequency diversity with simple implementation. The proposed schemes also provide full backward compatibility with the LTE system. Performance evaluation via simulation in a simplified LTE system environment corroborates that the proposed approach provides performance advantages in both downlink and uplink.
\end{abstract}

\section{INTRODUCTION}

In order to satisfy the fast growing demand of wireless services, the International Telecommunication Union (ITU) has started the standardization process of the next generation communication system (4G), namely IMT-Advanced systems [1]. IMT-Advanced systems establish a data rate requirement of $1 \mathrm{Gbps}$ at low mobile speeds (for fixed/nomadic terminals) and $100 \mathrm{Mbps}$ at high mobile speeds (for vehicles) as its target [2]. IEEE standardizes the enhancement of WiMAX, namely $802.16 \mathrm{~m}$ in order to meet the minimum requirement of IMTAdvanced, while 3rd Generation Partnership Project (3GPP) is drafting Long-Term Evolution Advanced (LTE-Advanced) standard to achieve the $4 \mathrm{G}$ requirement [3]. After the LTE standard is specified as Release 8 in the 3GPP [4], 3GPP starts the standardization process of LTE-Advanced as Release 10 with the following general requirements. First, LTE-Advanced will be an evolution of Release 8 LTE. Hence, distinctive performance gains from Release 8 LTE are required. Moreover, LTE-Advanced will satisfy all the relevant requirements for Release 8 LTE [5]. Second, full backward compatibility with Release 8 LTE is requested in LTE-Advanced. Thus, a set of user equipment (UE) for LTE-Advanced must be able to access Release 8 LTE networks, and LTE-Advanced networks must be able to support Release 8 LTE UEs. Third, LTEAdvanced should meet or exceed the IMT-Advanced minimum requirements within the ITU-R time plan [6].

In order to substantially increase data rate from LTE and keep backward compatibility, LTE-Advanced adopts the con- cept of carrier aggregation (CA), which uses multiple carrier components (CCs) in order to achieve the IMT-Advanced requirement, while each $\mathrm{CC}$ should also be backward compatible with LTE [7]. The CA approach introduces new implementation challenges, such as the handover procedures across multiple cell for CA-supported systems, how to set the guard band between different CCs, high PAPR and the limitation of the power amplifier (PA), etc. It can also provide opportunities for performance improvement.

Being a new approach, there are only limited works on CA. For instance, [8] provides uplink power control for CA, while [9] addresses cell search for CA and [10] presents a retransmission scheduling. Synchronization issue is addressed in [11] and methods for receiving and processing of CA signals are presented in [12] and [13]. In [14], a transmitter structure with configurable bandwidth for $\mathrm{CA}$ is proposed. All of these works address implementation issues for CA. Except being a mechanism for increasing data rate, other features or characteristics of CA have not been exploited yet for further performance improvement in the literature. In particular, multiple CCs can provide more frequency diversity, but this additional frequency diversity has not been exploited yet. The diversity can improve the signal to interference plus noise ratio (SINR) performance, which can help to reduce required transmit power. Consequently, this will lead to reduced peak output power and relaxed requirements on the PA, which is significant in CA-supported systems.

Traditionally, several mechanisms are used to exploit the frequency diversity. In [15]-[17], subcarrier and bit allocation helps to capture the frequency diversity. Typically, interleaved type (also called distributed type in LTE [18]) subcarrier allocation can capture frequency diversity. Interleaved coded modulation [19]-[21] can capture both time and frequency diversity. Precoding with subcarrier selection [22] and block spreading [23] can help to capture frequency diversity as well. All of these diversity exploitation methods consider single CC systems only.

The contribution of this paper is summarized as follows. Being an important mechanism to support high data rate while keeping backward compatibility with LTE, CA plays a crucial role for LTE-Advanced systems. The existing works on CA mainly address implementation issues, but exploitation of CAspecific features for further performance improvement has not 


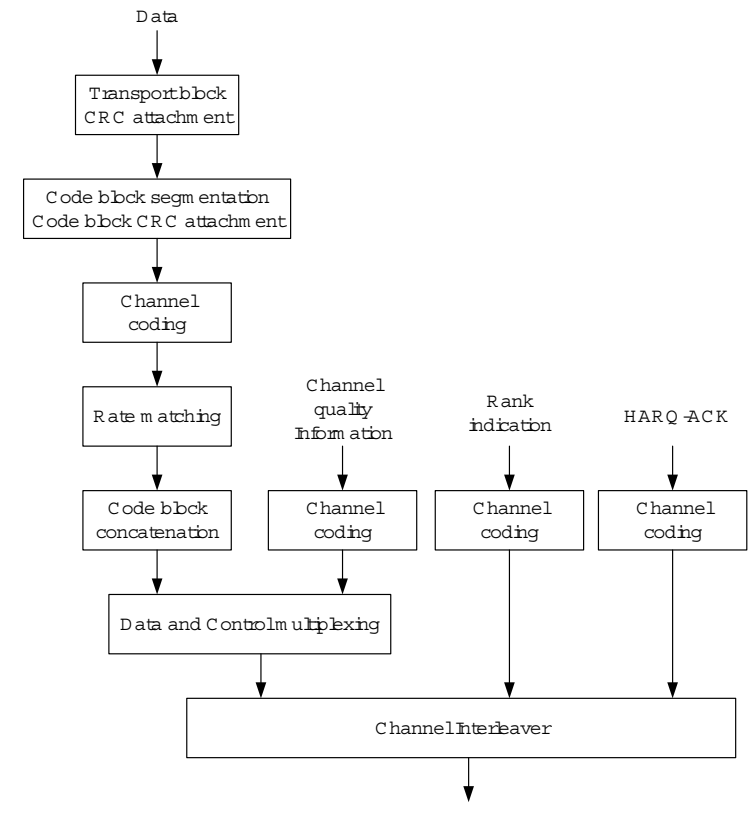

Fig. 1. Transport channel processing for UL-SCH

been addressed. We fill this gap and present how to exploit CA-specific frequency diversity. We introduce new interleaving schemes, which operate across the CCs for both downlink (DL) orthogonal frequency division multiple Access (OFDMA) as well as uplink (UL) single-carrier frequency-division multiple access (SC-FDMA) of LTE-Advanced systems. The new interleaving schemes adopt one-stage or two-stage structure while keeping compatibility with LTE and possessing low implementation complexity. The proposed schemes enhance both time and frequency diversity exploitation and improve the SINR performance.

The rest part of this paper is structured as below. In Section II, the processing in LTE is introduced. Based on the LTE structure, new interleavers are proposed in Section III. In Section IV, performance evaluation is presented. Finally, in Section V, the conclusion is given.

\section{Transport Channel Processing In LTE}

In LTE system, the transport channel processing procedures are different in DL and UL [24]. Here, we only focus on the shared channel, which is used to transmit data and some control information. The transport channel processing for UL shared channel (UL-SCH) is shown in Fig. 1. Data arrives at the coding unit in the form of a maximum of one transport block every transmission time interval (TTI). There are entirely seven steps as shown in Fig. 1. For frequency diversity exploitation, when the maximum bandwidth is used, interleaved coded modulation is the main mechanism to capture the frequency diversity. Consequently, channel coding module and rate matching module, which include internal sub-block interleaver are most relevant to frequency diversity exploitation. In channel coding module, turbo coding or tail

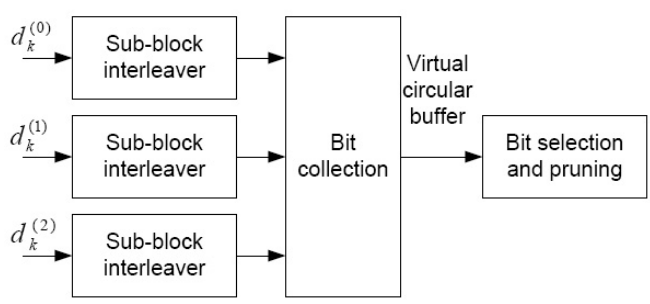

Fig. 2. The structure of rate matching module

biting convolutional coding with $1 / 3$ code rate is adopted. Both the coding structures have three output branches with corresponding codes denoted as $d_{k}^{(0)}, d_{k}^{(1)}, d_{k}^{(2)}$, respectively. In the rate matching module, there are three internal sub-block interleavers as shown in Fig. 2. The outputs of each branch $d_{k}^{(0)}, d_{k}^{(1)}, d_{k}^{(2)}$ in the channel coding module are the inputs to the sub-block interleavers, respectively. Since there are also control information (channel quality information (CQI), ranking information (RI) and hybrid ARQ-ACK (HARQACK)) transmitted by UL-SCH, a channel interleaver across the data and control information is applied in UL-SCH. This is a simple bit-vector (each bit vector corresponds to a modulation symbol) interleaver where bit vectors are written to a rectangular matrix row-by-row and read out column-bycolumn. Here, $C_{\mathrm{m}}$, the number of columns of the interleaving matrix, is set to be the same as $N_{\text {symb }}^{\mathrm{PUSCH}}$, the number of SCFDMA symbols in the current physical uplink shared channel (PUSCH) transmission sub-frame, which is given by

$$
N_{\text {symb }}^{\mathrm{PUSCH}}=2\left(N_{\mathrm{symb}}^{\mathrm{UL}}-1\right)-N_{\mathrm{SRS}},
$$

where $N_{\mathrm{SRS}}$ is equal to 1 if UE is configured to send PUSCH and sounding reference signal (SRS) in the same subframe. Otherwise, $N_{\mathrm{SRS}}$ is equal to 0 . Here, $N_{\mathrm{symb}}^{\mathrm{UL}}$ is the number of SC-FDMA symbols per slot in UL (one subframe includes two slots). When normal cyclic prefix (CP) is applied, $N_{\text {symb }}^{\mathrm{UL}}=7$. And in case of extended $\mathrm{CP}, N_{\mathrm{symb}}^{\mathrm{UL}}=6$. The number of modulation symbols in a subframe is $H$ and the number of rows of the matrix is

$$
R_{\mathrm{m}}=H / C_{\mathrm{m}} .
$$

Then we have the interleaving matrix as

$$
\left[\begin{array}{cccc}
Y_{0} & Y_{1} & \cdots & Y_{C_{\mathrm{m}}-1} \\
Y_{C_{\mathrm{m}}} & Y_{C_{\mathrm{m}}+1} & \cdots & Y_{2 C_{\mathrm{m}}-1} \\
\vdots & \vdots & \ddots & \vdots \\
Y_{\left(R_{\mathrm{m}}-1\right) \times C_{\mathrm{m}}} & Y_{\left(R_{\mathrm{m}}-1\right) \times C_{\mathrm{m}}+1} & \cdots & Y_{R_{\mathrm{m}} \times C_{\mathrm{m}}-1}
\end{array}\right] .
$$

For example, in the normal $\mathrm{CP}$ case, the length of each subframe is 14 symbols. Assuming that SRS is transmitted in the current subframe, we have $C_{\mathrm{m}}=2 \times(7-1)-1=11$.

The transport channel processing for DL shared channel (DL-SCH) is shown in Fig. 3. In DL, paging channel (PCH) and multicast channel $(\mathrm{MCH})$ have the same processing as in DL-SCH. The procedures of DL-SCH are quite similar to 


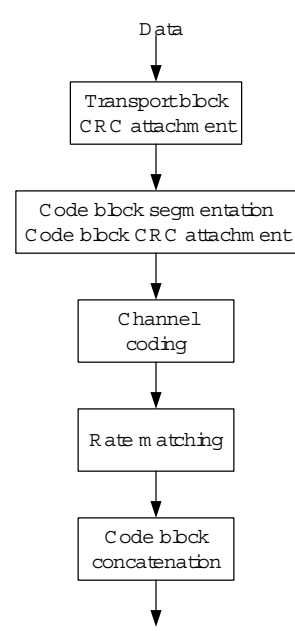

Fig. 3. Transport channel processing for DL-SCH

the UL-SCH. The only difference is that there is no channel interleaver used. Hence, from the view of frequency diversity exploitation, only the internal interleavers inside the rate-match module and turbo coding module help to capture the frequency diversity.

In LTE-Advanced system, each CC fulfills complete LTE features. More CCs will occupy more bandwidth. Intuitively, the information bit interleaved across the whole bandwidth will capture more frequency diversity than the case where each CC operates separately.

\section{ENHANCED FREQUENCY DIVERSITY EXPLOITATION IN CARRIER AGGREGATION}

In order to exploit the enhanced frequency diversity in CA without increasing too much complexity, we proposed several interleaver schemes with low complexity functioning across the different CCs for CA systems. If there are more potential frequency diversity provided by $\mathrm{CA}$, it should be captured by interleaving bits across CCs. Considering backward compatibility with LTE which is a significant requirement, we propose an interleaver revised from the channel interleaver adopted in LTE UL. In DL, the proposed interleaver is added over the $\mathrm{CCs}$, while in UL the proposed interleaver just takes place of the channel interleaver in LTE UL. The new interleavers are expected to exploit enhanced frequency and time diversity with the advantage of easy implementation.

\section{A. Two-Stage Interleaver Design}

We assume that there are entirely $N_{C}$ CCs. As shown in Fig. 4, the proposed interleaver includes two stages as

- Stage 1: Interleave (or multiplex) $N_{C}$ bit streams from $N_{C}$ CCs into one bit stream as

$$
\left[b_{1}(0), b_{2}(0), \ldots, b_{N_{\mathrm{C}}}(0), b_{1}(1), b_{2}(1), \ldots\right],
$$

where $b_{k}(n)$ is the $n$th bit from the $k$ th CC. This module refers to the Bit Multiplexing in Fig. 4.

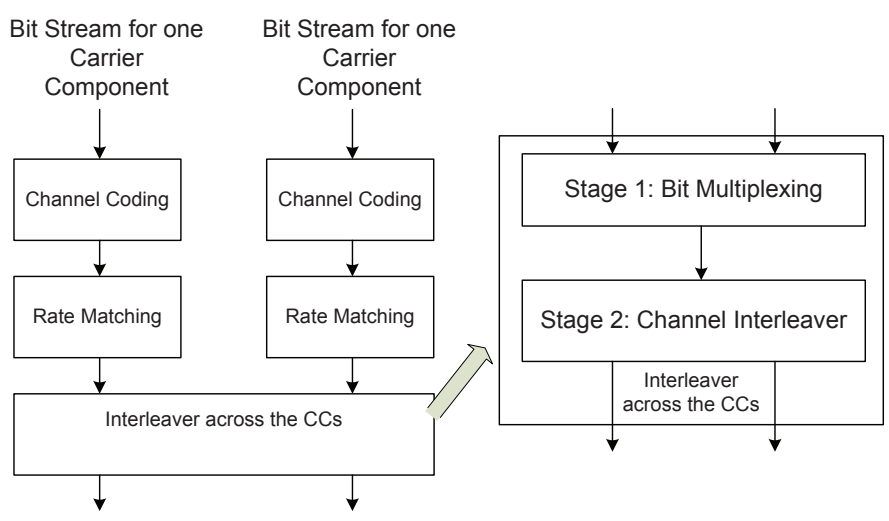

Fig. 4. Proposed transport channel processing in the performance evaluation

- Stage 2: Channel interleaver.

We propose two schemes of channel interleavers which are both modified from the channel interleaver used in LTE UL. In particular, when the number of CCs is one, the modified channel interleavers are both exactly the same as the channel interleaver used in LTE UL. Moreover, the data are only written into and read out from a matrix, which means the complexity of the proposed interleaver is very low.

1) Modified Channel Interleaver 1: The same as the channel interleaver in LTE UL, the modified channel interleaver functions as writing data into the interleaver matrix row-byrow and reading column-by-column. The difference is the size of the interleaver matrix and how to read from the interleaver matrix.

In the modified channel interleaver 1 , we use $C_{\mathrm{m}}$ as the number of columns, where $C_{\mathrm{m}}$ is set to $N_{\mathrm{symb}}^{\mathrm{PUSCH}}$ for $\mathrm{UL}$ and the number of symbols per subframe in DL-SCH for DL. Consequently, we can modify the interleaver matrix as

$\left[\begin{array}{cccc}Y_{0} & Y_{1} & \cdots & Y_{C_{\mathrm{m}}-1} \\ Y_{C_{\mathrm{m}}} & Y_{C_{\mathrm{m}}+1} & \cdots & Y_{2 C_{\mathrm{m}}-1} \\ \vdots & \vdots & \ddots & \vdots \\ Y_{\left(N_{\mathrm{C}} R_{\mathrm{m}}-1\right) \times C_{\mathrm{m}}} & Y_{\left(N_{\mathrm{C}} R_{\mathrm{m}}-1\right) \times C_{\mathrm{m}}+1} & \cdots & Y_{N_{\mathrm{C}} R_{\mathrm{m}} \times C_{\mathrm{m}}-1}\end{array}\right]$

We can also define each continuous $R_{\mathrm{m}}$ rows as a sub-matrix, which corresponds to a specific CC. After writing the data into the interleaver matrix, each $\mathrm{CC}$ reads data from its own sub-matrix.

2) Modified Channel Interleaver 2: The number of columns of the interleaver matrix used by the modified interleaver 2 is defined by $C_{\mathrm{m}}^{\prime}=N_{\mathrm{C}} \times C_{\mathrm{m}}$, while it is $C_{\mathrm{m}}$ for the interleaver in LTE UL. The interleaver matrix is modified from the channel interleaver in LTE UL as

$\left[\begin{array}{cccc}Y_{0} & Y_{1} & \cdots & Y_{C_{\mathrm{m}} N_{\mathrm{C}}-1} \\ Y_{\mathrm{m}_{\mathrm{m}} N_{\mathrm{C}}} & Y_{C_{\mathrm{m}} N_{\mathrm{C}}+1} & \cdots & Y_{2 C_{\mathrm{m}} N_{\mathrm{C}}-1} \\ \vdots & \vdots & \ddots & \vdots \\ Y_{\left(R_{\mathrm{m}}-1\right) \times C_{\mathrm{m}} N_{C}} & Y_{\left(R_{\mathrm{m}}-1\right) \times C_{\mathrm{m}} N_{C}+1} & \cdots & Y_{R_{\mathrm{m}} \times C_{\mathrm{m}} N_{C}-1}\end{array}\right]$, 
where $C_{\mathrm{m}}$ is defined as that in the modified channel interleaver 1.

When reading the data column-by-column, for $n$th CC it only reads columns with indexes $n, n+N_{C}, n+2 N_{C}, \ldots$ (in Matlab notation, the column indexes are $\left(n: N_{C}: C_{m}\left(N_{C}-\right.\right.$ 1) $+n)$ ).

Additionally, although we consider data only in this paper, the proposed interleavers can also be applied to the control information as follows. First, combine the control information bit streams from $N_{C}$ CCs into one bit stream. Second, cut the bit stream equally into $N_{C}$ subsequences and then interleave within each sub-matrix following the way used in LTE [24].

\section{B. One-Stage Interleaver}

We can also have a one-stage interleaver for CA-supported system by using either Stage 1 or Stage 2 with some adjustments. These one-stage interleavers can help to exploit the CA-specific diversity as well. The adjustments is described as follows. For Stage 1 (bit multiplexing), after multiplexing the bit streams from different CCs, we divide the combined bit sequence equally into $N_{C}$ subsequences. Each subsequence, containing multiplexed bits from all original CCs, is now transmitted on a CC. For the Stage 2, what we need to modify is the input part. Without the bit-multiplexing module, the bit sequences from different $\mathrm{CCs}$ are written into the interleaving matrix one sequence by one sequence. Then we can have a new interleaver for the CA-supported system.

Now, we have totally five candidate interleavers and use the following representation:

- Int1: modified channel interleaver 1 (one-stage),

- Int2: modified channel interleaver 2 (one-stage),

- SI: bit multiplexing (one-stage),

- S1+Int1: two-stage interleaver with the modified channel interleaver 1 as its channel interleaver,

- S1+Int2: two-stage interleaver with the modified channel interleaver 2 as its channel interleaver.

In UL, Int 1 without bit-multiplexing module is exactly the channel interleaver in LTE UL. And due to compatibility issue, interleaver $S 1$ can not be adopted. On the other hand, we find that when $N_{C}=1$, the two-stage interleavers $(S 1+I n t 1 \&$ $S 1+\operatorname{Int2}$ ) become the same as the channel interleaever used in LTE UL. Thus, the backward compatiblity with LTE UL is automatically achieved by the two-stage interleavers.

For DL, all these five interleavers can be used. And when $N_{C}=1$, the interleaever $S 1$ reduces to a module which only introduces a delay. Thus, the compatibility with LTE DL can be automatically achieved by $S 1$.

Except those interleavers which can automatically satisfy the compatibility requirement, we need a conditional module which judges whether $N_{C}=1$. If $N_{C}=1$, then it should be switched to LTE scheme to assure the compatibility. From compatibility perspective, $S 1+I n t 1$ and $S 1+I n t 2$ have some advantages in UL, and $S 1$ has some advantages in DL.

In terms of the complexity, obviously $S 1$ has the lowest complexity in these proposed interleavers. However, even the
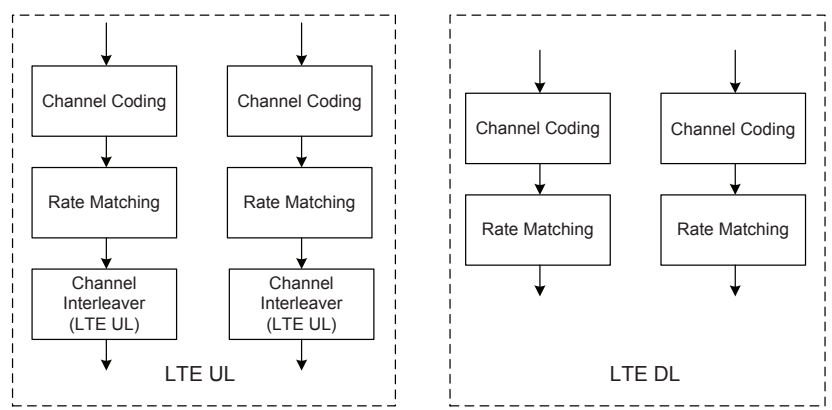

Fig. 5. LTE transport channel processing in the performance evaluation

two-stage interleavers, which have the highest complexity among these interleavers, have the complexity of $O\left(H N_{C}\right)$. This means that the proposed interleavers are all with low complexity.

\section{Performance Evaluation}

\section{A. Simulation Parameters}

We compare the performances due to the frequency diversity exploitation of the proposed schemes and LTE scheme in this section. Since interleaved coded modulation (including channel coding module, rate matching module and interleaver module) is the main mechanism to capture the frequency and time diversity in this case, we simplified the simulation model for the proposed approach as in Fig. 4 so that we can accelerate the simulation without sacrificing the accuracy. For comparison, we use the same simplified model to simulate the performance of LTE. The structures of UL and DL of LTE are simplified as in Fig. 5.

We consider the case of two independent (non-contiguous) CCs. Each CC has the bandwidth of $20 \mathrm{MHz}$, in which case 1200 subcarriers are used with 2048 DFT size. The channel model from project WINNER II [25] is used. The operating scenario is assumed to be urban macro-cell (UMa). As defined in [24], turbo code is applied as the coding scheme for shared channel. Since the convolutional code is also adopted in some other channel in LTE [24], we also evaluate the performance of $[133,177,165]$ convolutional code. We suppose $N_{\mathrm{SRS}}=0$, then according to (1) we have $N_{\text {symb }}^{\mathrm{PUSCH}}=12$ in UL. For DL, we assume that entirely two OFDM symbols are occupied by physical downlink control channel (PDCCH) and SRS. Then $C_{\mathrm{m}}$ is also 12 in DL. For simplicity, we only generate the data in PUSCH and PDSCH in our simulation. Then we have the length of each subframe in both UL and DL equal to 12 symbols.

QPSK is used for both DL and UL. In DL simulation, OFDMA is applied, while SC-FDMA is adopted in UL. For the turbo code, Log-MAP algorithm is used in the turbo decoder for both UL and DL. For the convolutional code, Viterbi decoder with truncated maximum likelihood (ML) criterion is adopted in DL, while a frequency-domain Minimum meansquare error (MMSE) equalizer followed by a truncated Viterbi decoder is adopted in UL. 


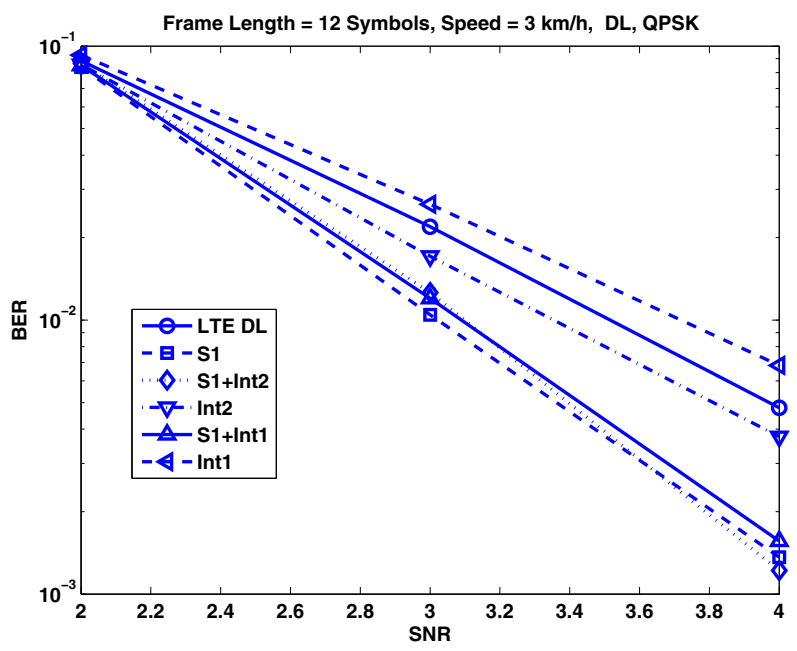

Fig. 6. Turbo code performance in DL at the speed of $3 \mathrm{~km} / \mathrm{h}$

\section{B. Simulation Results}

The bit-error rate (BER) performances for the turbo code at mobile speed of $3 \mathrm{~km} / \mathrm{h}$ in DL is shown in the Fig. 6 . We can observe that there exists additional frequency diversity in CA systems and this diversity can be captured by interleavers across the CCs. The two-stage interleavers $(S 1+I n t 1$ and $S 1+I n t 2)$ and the interleaver $S 1$ have similar performances, which outperform the intereleaver of LTE DL substantially. Additionally, we can observe that the slopes of the proposed interleaver performance curves are obviously larger than that of LTE DL, which means more diversity is captured by the proposed interleavers.

For the convolutional code, the BER performance at mobile speed of $3 \mathrm{~km} / \mathrm{h}$ in DL is shown in Fig. 7. The performances of the proposed interleavers are better than the standard LTE DL as well. Especially, the proposed two-stage interleaver $S 1+$ Int 1 outperforms all the rest cases. And comparing the slope differences between the convolutional code and the turbo code, we find that the proposed interleavers can capture more diversity for the turbo coding than the convolutional coding. Since in LTE system, the turbo code is adopted by more channels including the shared channel which we focus on, the proposed interleavers are attractive to the CA-supported systems.

As LTE-Advanced systems support high mobility service, we also evaluate the BER performance at the mobile speed of $120 \mathrm{~km} / \mathrm{h}$. Due to limited space, we only provide the BER performance of the turbo code at the speed of $120 \mathrm{~km} / \mathrm{h}$ as shown in Fig. 8. The result is quite similar to those in Fig. 6 , which illustrates that our proposed schemes also work well in high mobile speed scenarios. Comprehensively considering BER, complexity and compatibility issues, we conclude that the proposed interleaver $S 1$ is the best choice for the DL shared channel in CA-supported systems.

For UL, the performances at the mobile speed of $3 \mathrm{~km} / \mathrm{h}$ for the turbo code and the convolutional code are also given

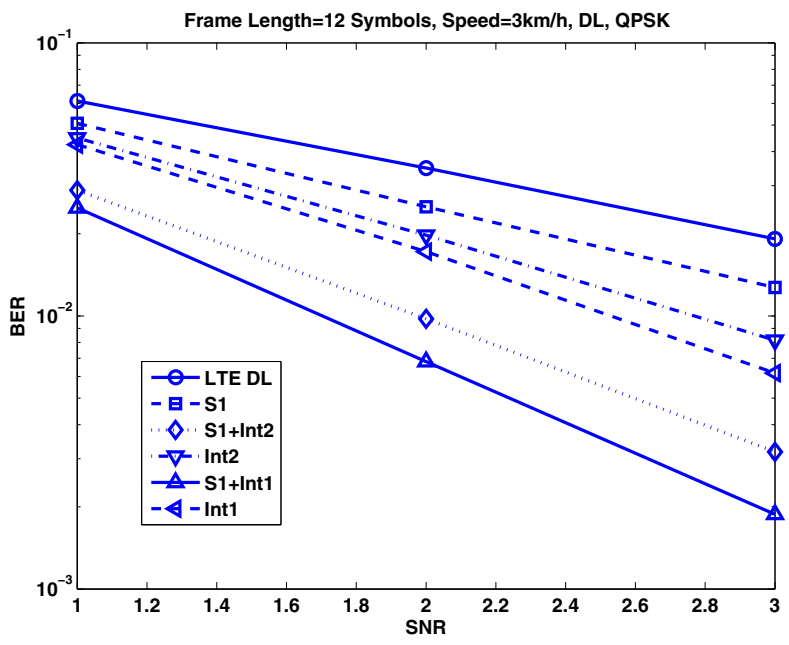

Fig. 7. Convolutional code performance in DL at the speed of $3 \mathrm{~km} / \mathrm{h}$

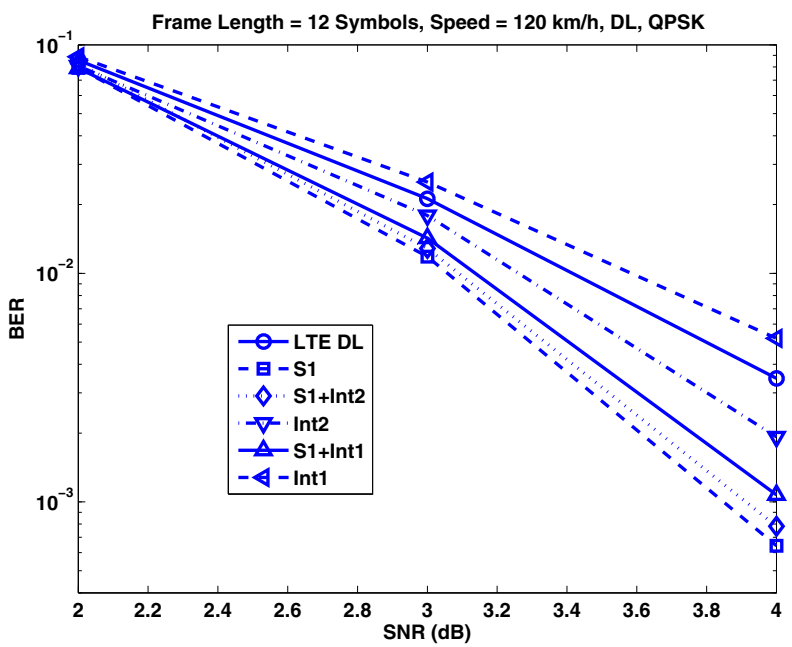

Fig. 8. Turbo code performance in DL at the speed of $120 \mathrm{~km} / \mathrm{h}$

in Figs. 9 and 10, respectively. The proposed interleavers in these cases are also able to capture the additional frequency diversity brought in by the CA technique. For the turbo coding, the interleavers $S 1+\operatorname{Int} 2$ and Int 2 outperform the rest, while all the proposed interleavers have better performance than the standard LTE UL interleaver. For the convolutional code, the interleavers $S 1+$ Int1 outperforms the other cases. Also, the proposed interleavers show more advantages (larger slopes) in the turbo coding than the convolutional coding. We also evaluate the BER performance at the speed of $120 \mathrm{~km} / \mathrm{h}$ (due to space limitation, we do not include the results here), the performances are still similar to those at the speed of $3 \mathrm{~km} / \mathrm{h}$. Comprehensively, $S 1+\operatorname{Int} 2$ is the best choice in UL shared channel in CA-supported systems.

\section{CONCLUSIONS}

As LTE-Advanced standardization adopts CA as a means to meet data rate requirements of IMT-Advanced system 


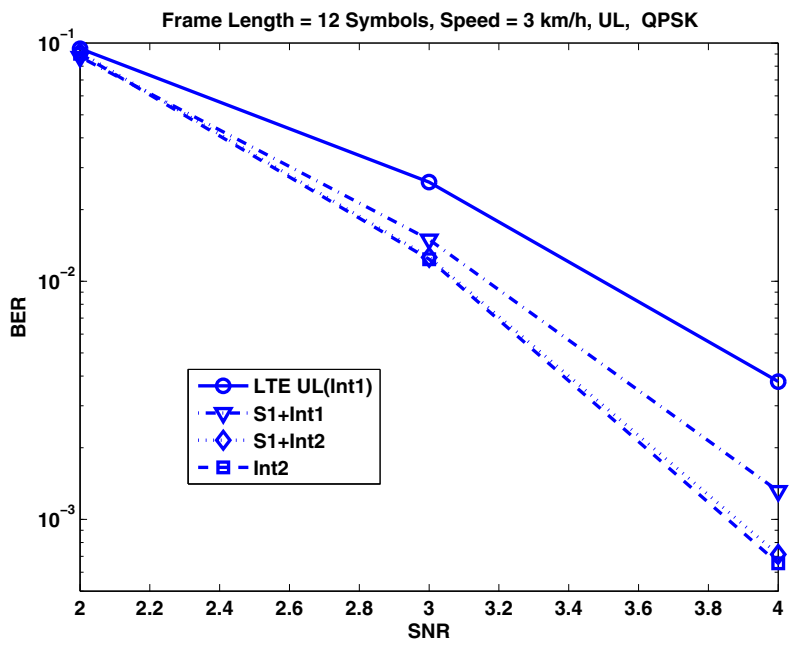

Fig. 9. Turbo code performance in UL at the speed of $120 \mathrm{~km} / \mathrm{h}$

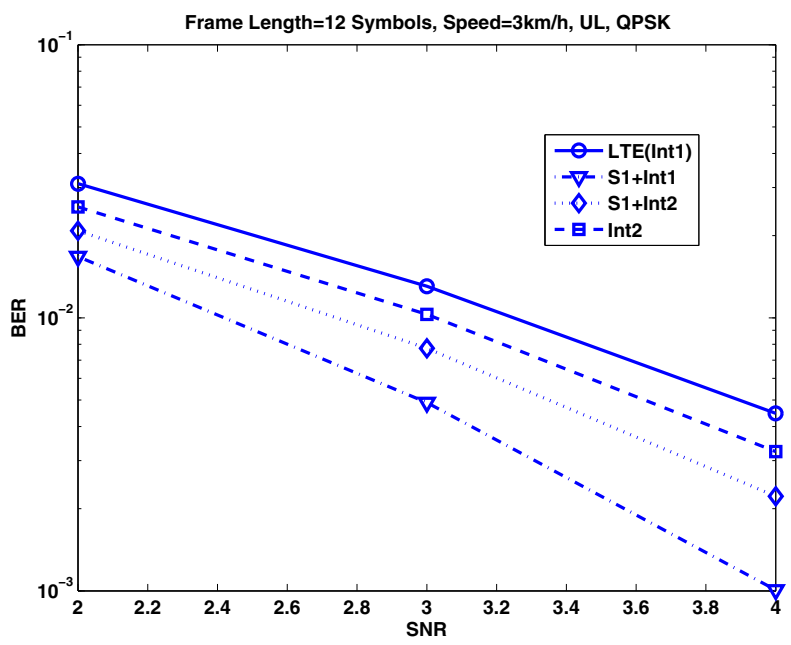

Fig. 10. Convolutional code performance in UL at the speed of $3 \mathrm{~km} / \mathrm{h}$

specification, it becomes important to investigate features of CA and exploit them for further improvement. This paper proposed new two-stage and one-stage interleavers with low complexity to explore and capture additional frequency diversity brought in by the CA. The proposed interleavers provide several advantages such as low implementation complexity, enhanced frequency and time diversity exploitation, and backward compatibility with LTE systems. Our performance evaluation shows a significant BER performance improvement of the proposed approach over the standard LTE approach, and hence the proposed approach can serve as a viable performance enhancement technique for LTE-Advanced systems.

\section{REFERENCES}

[1] ITU-R Rec. M. 1645, "Framework and overall objectives of the further deverlopment of IMT-2000 and systems beyond IMT-2000," 2003.
[2] WP5D, "Draft report on requirements related to technical perforamnce for IMT-Advanced radio interface(s) [IMT.TECH]," Working Party 5D Sub-Working Group Radio Aspects, 2nd Meeting, Dubai, United Arab Emirates, July 2008

[3] 3GPP, LTE-Advanced, "Requirements for further advancements for EUTRA"(Release 8), 3GPP TR 36.913 v8.0.1, March 2009.

[4] 3GPP, Long-Term Evolution (LTE), "LTE Physical layer-general description”(Release 8), 3GPP TS 36.211 v8.3.0, Feb. 2009.

[5] 3GPP, Long-Term Evolution, " Requirements for Evolved UTRA (EUTRA) and Evolved UTRAN (E-UTRAN)", 3GPP TR 25.913 v7.3.0, March 2006.

[6] M. Sawahashi, Y. Kishiyama, H. Taoka, M. Tanno and T. Nakamura, "Broadband radio acess: LTE and LTE-Advanced," ISPACS, Dec. 2009, pp. 224-227.

[7] 3GPP, Long-Term Evolution (LTE), "Further Advancements for E-UTRA: Physical Layer Aspects"(Release 9), 3GPP TR 36.814 v1.2.1, June 2009.

[8] Y. Perets, E. Melzer, and I. Bergel, "Uplink power control in aggregated spectrum systems," US 2010/0015967 A1, Jan. 21, 2010.

[9] B. Lindoff, E. Dahlman, Y. Jading, and S. Parkvall "Identifying multicomponent carrier cells," US 2009/0316659 A1, Dec. 24, 2009.

[10] A. Rosenqvist, M. Kamuf, and B. Lindoff, "Method and apparatus for retransmission scheduling and control in multi-carrier wireless communication networks," US 2009/0279480 A1, Nov. 12, 2009.

[11] B. Lindoff, B. Bernhardsson, and F. Nordstrom, "Time-error and frequency-error correction in a multi-carrier wireless communications system," US 2009/0258628 A1, Oct. 15, 2009.

[12] F. Nordstrom, N. Andgart, and B. Lindoff, "Wireless communication methods and receivers for receiving and processing multiple component carrier signals," US 2009/0257517 A1, Oct. 15, 2009.

[13] B. Lindoff, N. Andgart, and F. Nordstrom, "System and method of receiving and processing multicommunication signals," US 2009/0257533 A1, Oct. 15, 2009

[14] E. N. Onggosanusi, A. G. Dabak, B. Varadarajan, R. Chen, and T. Muharemovic, "Backward compatible bandwidth extension," US 2009/0268831 A1, Oct. 29, 2009.

[15] H. Minn and C.-C. Chong, "User resource structure design with enhanced diversity for OFDMA in time-varying channels" IEEE WCNC, April 2009, pp.646-651.

[16] B. Bai, W. Chen, Z. Cao and K. B. Letaief, "Achieving high frequency diversity with subcarrier allocation in OFDMA systems", IEEE GLOBECOM, Nov. 2008, pp.1-5.

[17] C. Y. Wong and R. S. Cheng "Multiuser OFDM with adaptive subcarrier, bit, and power allocation", IEEE J. Sel. Areas Commun., vol. 17, no. 10, Oct. 1999, pp. 1747-1758.

[18] 3GPP, Long-Term Evolution (LTE), "Physical Channels and Modulation"(Release 8), 3GPP TS 36.211 v8.6.0, March 2009.

[19] D. Huang, K. B. Letaief and J. Lu, "Bit-interleaved time-frequency coded modulation for OFDM systems over time-varying Channels", IEEE Trans. Commun., vol. 53, no. 7, July 2005, pp. 1191-1199.

[20] G. Caire, G. Taricco and E. Bigloeri, "Bit-Interleaved Coded Modulation", IEEE Trans. Info. Theory, vol. 44, no. 3, MAY 1998, pp. 927-946.

[21] E. Akay and E. Ayanoglu, "Bit-interleaved Coded Modulation: Low Complexity Decoding," IEEE VTC, May 2004, p.328-332.

[22] C. R. N. Athaudage, J. Wang and A. D. S. Jayalath, "An efficient framework to exploit frequency diversity in OFDM: precoding with adaptive subcarrier selection", IEEE PIMRC, Sep. 2006, pp. 1-5.

[23] M. L. McCloud, "Optimal binary spreading for block OFDM on multipath fading channels", IEEE WCNC, vol. 2, March 2004, pp. 965970.

[24] 3GPP, Long-Term Evolution (LTE), "Multiplexing and channel coding"(Release 9), 3GPP TS 36.212 v9.0.0, Dec. 2009.

[25] Wireless World Initiative New Radio-WINNER II, "Matlab SW documentation of WIM2 model” IST-4-027756, v1.0, Aug. 2008. 